\title{
Astrogliosis in Amyotrophic Lateral Sclerosis: Role and Therapeutic Potential of Astrocytes
}

\author{
Marcelo R. Vargas* and Jeffrey A. Johnson* $* \$ \S$ \\ $*$ Division of Pharmaceutical Sciences, ${ }^{\dagger}$ Waisman Center, ${ }^{\ddagger}$ Molecular and Environmental Toxicology Center, ${ }^{\S}$ Center for \\ Neuroscience, University of Wisconsin, Madison, Wisconsin, 53705
}

Summary: Amyotrophic lateral sclerosis (ALS) is a fatal disorder characterized by the progressive loss of motor neurons. Although the molecular mechanism underlying motor neuron degeneration remains unknown; non-neuronal cells (including astrocytes) shape motor neuron survival in ALS. Astrocytes closely interact with neurons to provide an optimized environment for neuronal function and respond to all forms of injury in a typical manner known as reactive astrogliosis. A strong reactive astrogliosis surrounds degenerating motor neurons in ALS patients and ALS-animal models. Although reactive astrogliosis in ALS is probably both primary and secondary to motor neuron degeneration; astrocytes are not passive observers and they can influence motor neuron fate. Due to the important functions that astrocytes perform in the central nervous system; it is of key importance to understand how these functions are altered when astrocytes become reactive in ALS. Here; we review the current evidences supporting a potential toxic role of astrocytes and their viability as therapeutic targets to alter motor neuron degeneration in ALS. Key words: Astrogliosis, amyotrophic lateral sclerosis, excitotoxicity, oxidative stress, death receptors, glutathione.

\section{INTRODUCTION}

Astrocytes represent the largest cell population in the CNS. Once regarded as the element that fills the space not occupied by neurons, the last 20 years of research have identified a plethora of essential functions that these cells perform in the healthy nervous system. Traditionally, astrocytes have been classified as protoplasmic (grey matter) and fibrous (white matter), but astrocytes are a heterogeneous population, and other types of astrocytes can be identified in specific regions (i.e., the radial glia of the retina or the velate astrocytes of the olfactory bulb). In addition, special types of astrocytes are found exclusively in certain species, such as the interlaminar astrocytes and varicose projection astrocytes in the cortex of higher-order primates. ${ }^{1}$ In contrast to the seemingly stellate appearance of astrocytes conferred by the staining for the intermediate filament glial fibrillary acidic protein (GFAP), ${ }^{2}$ protoplasmic astrocytes exhibit an intricate bushy or spongiform morphol-

Address correspondence and reprint requests to: Jeffrey A. Johnson, Ph.D., School of Pharmacy, 6125 Rennebohm Hall, 777 Highland Avenue, University of Wisconsin, Madison, WI 53705. E-mail: jajohnson@pharmacy.wisc.edu. ogy due to many fine membrane processes that become evident when cells are filled with dye. These fine processes have minimal spatial overlap with the processes of other astrocytes, and they establish exclusive territories for individual astrocytes. ${ }^{3}$ In the human cortex, a single astrocyte enwraps more than 1 million synapses, and most, if not all, have at least one process with end feet surrounding a blood vessel. This particular arrangement not only influences the structural architecture of the brain, but it also has the capacity to define functional compartments, ${ }^{4}$ and it places astrocytes in a key position to provide structural, metabolic, and trophic support to neurons. Astrocytes are a reservoir of glycogen, and by controlling ionic and osmotic homeostasis, new evidence suggests they are key players in the global and regional control of cerebral blood flow in response to neuronal activity. ${ }^{5}$ Astrocytes also play a crucial role in the antioxidant defenses of the brain, determining the vulnerability of neurons to noxious stimuli. ${ }^{6}$ In addition, astrocytes influence neuronal excitability by removing neurotransmitters, such as glutamate form the synaptic cleft, but they may also integrate and process synaptic information in what has been called the "tripartite synapse."7

In the CNS of higher vertebrates, astrocytes respond to all forms of injury, such as trauma, infection, ischemia, 
and neurodegenerative processes in a typical manner known as reactive astrogliosis. ${ }^{8,9}$ Reactive astrocytes are characterized by the hypertrophy of cellular processes, upregulation of GFAP and vimentin, and re-expression of nestin. This is accompanied by nuclei hypertrophy and altered expression of many others proteins, such as proteases, growth factors, and cytokines. ${ }^{10,11}$ In some paradigms, a considerable proportion of quiescent astrocytes resume proliferation with injury, and they contribute to the generation of reactive astrocytes, ${ }^{12}$ but the degree of proliferation of GFAP-expressing cells vary with the nature and severity of the injury. ${ }^{9,13}$ How astrogliosis affects neuronal physiology and survival is most probably injury-specific and will be determined by the impact of the phenotypic change in the normal astrocytic functions, as well as by the potential acquired new characteristics. In addition, reactive astrocytes interact in a complex manner with microglia and can exert pro-inflammatory and anti-inflammatory effects. Because reactive astrogliosis occurs in all major neurodegenerative diseases, it has long been suggested to be a nonspecific response of glial cells to injury, and it is often not considered to be a primary pathogenic element. However, recent evidence indicates that in amyotrophic lateral sclerosis (ALS), astrocytes are able to influence the course of the disease and are a potential therapeutic target.

\section{ASTROGLIOSIS IN ALS}

ALS is the most common adult-onset motor neuron disease, caused by the progressive degeneration of motor neurons in the spinal cord, brainstem, and motor cortex. ${ }^{14}$ The etiology of most ALS cases remains unknown (sporadic ALS); however, 10\% are inherited in a dominant manner (familial ALS, [FALS]). Both forms of ALS share the same pathological features, including progressive muscle weakness, atrophy, and spasticity. Usually, death occurs within 3 to 5 years from onset. Approximately $10 \%$ to $20 \%$ of FALS is caused by a toxic gainof-function induced by mutations in the enzyme $\mathrm{Cu} / \mathrm{Zn}$ superoxide dismutase (SOD1). ${ }^{15}$ Rodents overexpressing mutated forms of FALS-linked human SOD1 (hSOD1) generally develop progressive motor neuron degeneration resembling many aspects of ALS. ${ }^{16,17}$ In ALS patients, a reactive astrogliosis surrounds both upper and lower motor neurons, ${ }^{18-21}$ although cortical astrogliosis may not be ALS-specific and arise from contemporary pathologies due to long hypoxic distress in terminal patients. ${ }^{22}$ Reactive astrocytes are also found in the region where the descending fibers of cortico-spinal tracts enter the grey matter. ${ }^{21}$ Reactive astrocytes in ALS show increased immunoreactivity for GFAP and the calciumbinding protein $\mathrm{S} 100 \beta$ and express inflammatory markers, such as cyclo-oxygenase-2 (COX-2), inducible nitric oxide synthase (iNOS), and neuronal nitric oxide syn- thase. Increase in GFAP-immunoreactive astrocytes is particularly notable in the grey matter of the spinal cord ventral horn, where normally astrocytes express/stain for GFAP at very low levels. In addition, cytoplasmic hyaline inclusions and markers of oxidative and nitrative stress accompany astrocyte pathology. ${ }^{22,23}$

In mutant hSOD1 transgenic mice, a similar pattern of reactive astrogliosis has been described, ${ }^{17,24-26}$ although the time course of astrogliosis, relative to motor neuron degeneration and the onset of symptoms, seems to vary in different models. Motor neuron loss in animals expressing hSOD $1^{\mathrm{G} 37 \mathrm{R}}$ typically starts around 15 weeks of age, whereas reactive astrogliosis in the ventral horn of the spinal cord is evident as early as 5 weeks of age. ${ }^{24}$ Astrogliosis also becomes more prominent as the disease advances, accompanying the vacuolar degeneration of mitochondria in the motor neurons. ${ }^{24}$ In hSOD1 ${ }^{\mathrm{G} 85 \mathrm{R}}$ mice, initial indicators of disease are astrocytic inclusions that stain for SOD1, and ubiquitin and SOD1containing aggregates in motor neurons. Reactive astrogliosis becomes evident before disease onset and escalates markedly as the disease progresses, concomitant with a decrease in the glial glutamate transporter (GLT1), ${ }^{25}$ thus suggesting that a direct effect on astrocytes mediates at least some of the damage caused by hSOD1 mutants.

In contrast, in the most widely used mice model $\left(\mathrm{hSOD} 1^{\mathrm{G} 93 \mathrm{~A}}\right.$ ), reactive astrogliosis develops at the onset of symptoms after motor neuron loss is observed, ${ }^{26,27}$ and this is accompanied by microglia infiltration. Reactive astrocytes also express iNOS and display nitrotyrosine immunoreactivity. ${ }^{28,29}$ In symptomatic hSOD1 ${ }^{\mathrm{G} 93 \mathrm{~A}}$ animals, reactive astrocytes extend processes toward neuronal vacuoles and wrap around them, ${ }^{27}$ indicating that either as a primary or as a reactive response, astrocytes actively participate in the neuronal degeneration process. A robust astrogliosis can also be observed in the spinal cord of symptomatic hSOD $1^{\mathrm{L} 126 \mathrm{Z}}$ mice, ${ }^{30}$ as well as in two other models expressing the experimental mutations hSOD1 ${ }^{\mathrm{H} 46 \mathrm{R} / \mathrm{H} 48 \mathrm{Q}}$ and hSOD1-Quad. ${ }^{31,32}$ Finally, astrogliosis precedes both motor neuron loss and clinical signs of diseases in hSOD $1^{\mathrm{G} 93 \mathrm{~A}}$ and $\mathrm{hSOD} 1^{\mathrm{H} 46 \mathrm{R}}$ rats, ${ }^{17,33}$ and this coincides with early vacuolization of the neuropil and a striking focal loss of the GLT1-glutamate transporter in the ventral horn. Therefore, changes in astrocytes are a prominent and generally preclinical abnormality in ALS-models, supporting the idea that molecular targets within astrocytes can be damaged by the expression of mutant SOD1.

\section{TOXICITY OF MUTANT SOD1 AND THE ORIGIN OF ASTROGLIOSIS}

Several hypotheses, including oxidative stress, glutamate excitotoxicity, decreased trophic support, formation 
of high molecular weight aggregates, defective axonal transport, and mitochondrial dysfunction have all been proposed to explain the toxic effect of mutant SOD1. ${ }^{34-37}$ Although the molecular mechanism underlying the relative selective death of motor neurons remains unknown, in animal models the toxicity requires the expression of mutant hSOD1 in non-neuronal cells, as well as in motor neurons. Initial experiments showed that transgenic mice expressing mutated forms of hSOD1 exclusively in astrocytes ${ }^{38}$ or neurons ${ }^{39,40}$ fail to develop overt motor neuron disease. However, it has been shown recently that mice overexpressing $\mathrm{hSOD} 1{ }^{\mathrm{G} 93 \mathrm{~A}}$ under the Thy 1.2 expression cassette at much higher levels develop motor neuron degeneration. ${ }^{41}$ Nevertheless, this model exhibits a very late and diffuse onset of motor symptoms relative to transgenic lines expressing $\mathrm{hSOD} 1^{\mathrm{G} 93 \mathrm{~A}}$ ubiquitously. Interestingly, in this later study, the expression of mutant hSOD1 restricted to neurons caused astrogliosis limited to regions with high levels of neuronal degeneration. ${ }^{41}$ Along with the general observation that astrogliosis parallels neuronal degeneration and reaches its highest level at the end stage of the disease, this finding suggests that astrogliosis responds to the ongoing neuronal degeneration.

However, this does not mean that astrogliosis is only secondary to motor neuron degeneration, because the expression of mutant mouse SOD1 under the control of the GFAP-promoter (GFAP-mSOD1 ${ }^{\mathrm{G} 86 \mathrm{R}}$ ) is sufficient to cause astrogliosis in the absence of motor neuron pathology. ${ }^{38}$ Yet, astrogliosis never reaches the levels observed in terminal animals expressing mutant hSOD1 ubiquitously, and it is not observed in young GFAPmSOD ${ }^{\mathrm{G} 86 \mathrm{R}}$ animals. Because the capacity of mutant hSOD1 to induce disease is proportional to its expression level,${ }^{16,41}$ it would be interesting to determine whether mutant hSOD1 expression in astrocytes, at higher levels than the ones achieved in the original study ${ }^{38}$ may have a detrimental effect on motor neuron survival in vivo. Altogether, the most probable scenario is that astrogliosis is both primary and secondary to neuronal damage, with reactive astrocytes actively participating in the neuronal degeneration process. It is noteworthy that most of the data arises from animal models of FALS, and that in ALS patients, other factors such as aging could contribute to the observed astrogliosis. ${ }^{23}$

The classic definition of reactive astrogliosis includes cellular proliferation; however, the degree of proliferation appears quite variable depending on the type of injury studied and the techniques used. The targeted expression of the herpes simplex virus thymidine kinase under the control of the GFAP-promoter renders dividing astrocytes vulnerable to the antiviral drug ganciclovir resulting in the specific ablation of astrocytes that have strong GFAP promoter activity and are undergoing proliferation. This model has been used to demonstrate that the selective ablation of proliferating GFAP + astrocytes does not affect the course of disease in hSOD1G93A mice. ${ }^{42}$ However, the overall cell proliferation and total astrogliosis were not significantly reduced, indicating that in this ALS model the contribution of proliferating GFAP + cells to astrogliosis is minimal. In addition, it suggests that the majority of new astrocytes are derived from glial progenitors, probable Olig2 $+/ \mathrm{NG} 2+$ glial progenitors, and not GFAP+-proliferating cells. ${ }^{13}$

A strong argument in favor of the participation of non-neuronal cells in ALS pathology came from the generation of chimeric animals. ${ }^{43}$ In these mice, the degeneration of motor neurons expressing mutant hSOD1 was delayed when surrounded by wild-type non-neuronal cells, whereas wild-type motor neurons surrounded by cells expressing mutant hSOD1 developed degenerative changes. The relative contribution of mutant hSOD1 in each cell type was later suggested in gene excision experiments by cell-specific Cre recombinase expression and cell replacement strategies. The proposed noncellautonomous mechanism of the disease suggests that although the expression of the mutant enzyme in motor neurons affects disease onset, ${ }^{44-46}$ the expression within the glial compartment influences disease progression. ${ }^{44,47,48}$ The damage exerted by mutant hSOD1 in different cell types, and its contribution to motor neuron degeneration, has been extensively reviewed. ${ }^{49-51}$ The following sections will focus on the current evidence that supports an active role of astrocytes in ALS and their potential therapeutic applications (FIG. 1).

\section{FUNCTIONAL ALTERATIONS OF ASTROCYTES IN ALS}

\section{Excitotoxicity}

One of the long-standing hypotheses for motor neuron degeneration in ALS proposes that a selective impairment in glial glutamate transport causes the accumulation of excitotoxic levels of extracellular glutamate. Glutamate released in the synaptic cleft is taken up by astrocytes through sodium-dependent excitatory amino acid transporters EAAT1 (GLAST) and EAAT2 (GLT1). If glutamate is not removed, sustained elevation of neuronal intracellular calcium levels through permissive glutamate receptors initiates a cascade of events that leads to cell death. Many ALS patients have elevated glutamate levels in the cerebrospinal fluid ${ }^{52,53}$ and a decrease in EAAT2 expression in the motor cortex and spinal cord. ${ }^{54}$ In addition, in the spinal cord of end-stage hSOD1 ${ }^{\mathrm{G} 85 \mathrm{R}}$ transgenic mice there is a $50 \%$ decrease in the levels of GLT1 protein, ${ }^{25}$ whereas in the in hSOD $1^{\mathrm{G} 93 \mathrm{~A}}$ transgenic rats, there is a focal loss of transporters around motor neurons in presymptomatic animals and almost a complete loss at the end-stage of the disease. ${ }^{26}$ However, in hSOD $1^{\mathrm{G} 93 \mathrm{~A}}$ mice, changes in GLT1 levels in the 


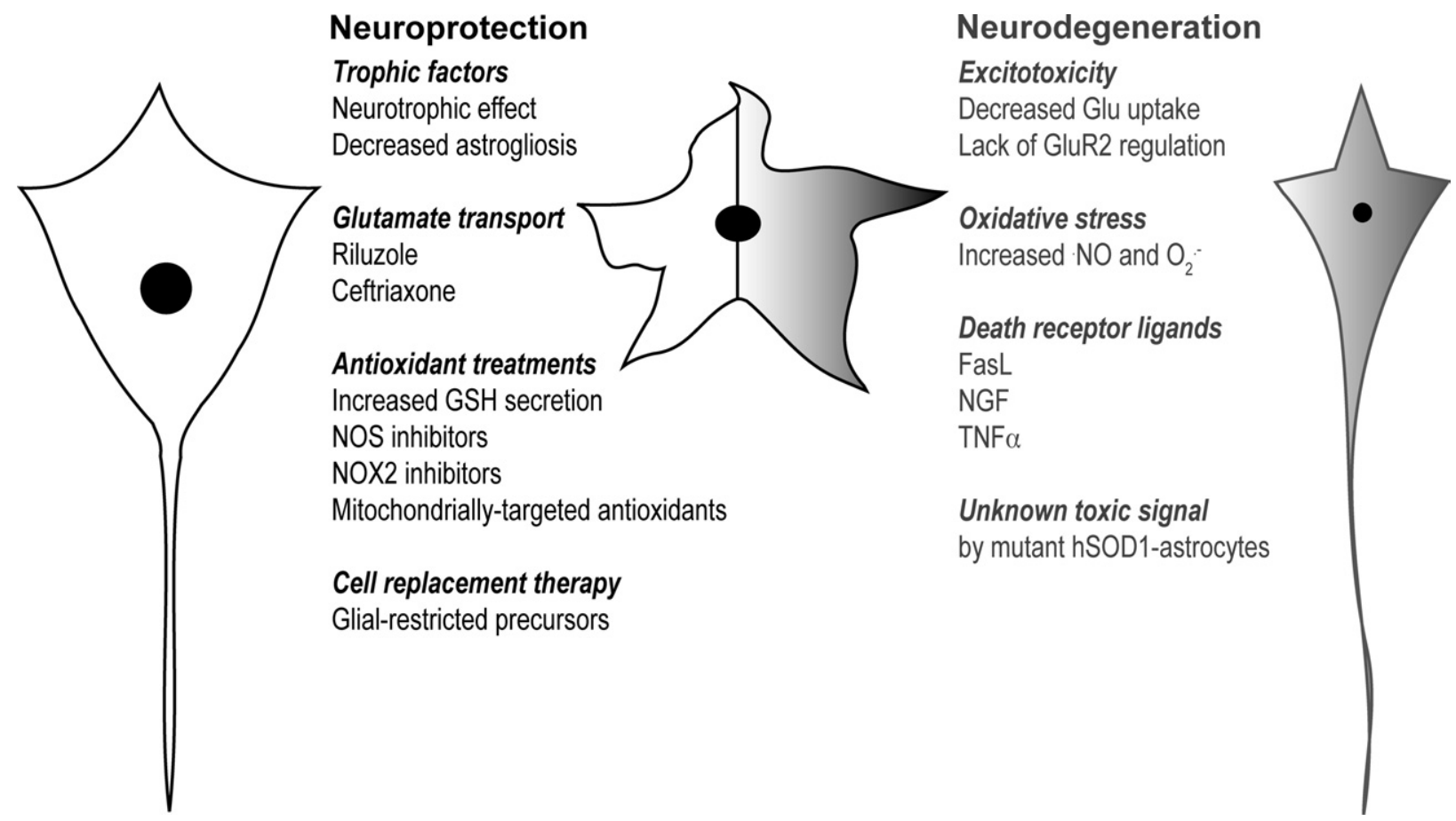

FIG. 1. The diagram summarizes the role of astrocytes in amyotrophic lateral sclerosis (ALS) and some therapeutic strategies. (See text for details.) FasL = FasL-Fas ligand; Glu = glutamate; GluR2 = glutamate receptor subunit 2; GSH = $\gamma$-I-glutamyl-I-cysteinylglycine; hSOD-1 = human superoxide dismutase 1; NGF = nerve growth factor; NOS = nitric oxide synthase; NO = nitic oxide; NOX2 = NADPH oxidase; TNF $\alpha=$ tumor necrosis factor $\alpha$.

spinal cord only occur after the initiation of symptoms and motor neuron loss is observed. ${ }^{55}$ Interestingly, overexpression of EAAT2 under the control of the human GFAP-promoter in hSOD1 ${ }^{\mathrm{G} 93 \mathrm{~A}}$ improves motor performance, but does not delay the onset of paralysis nor prolong survival. ${ }^{56}$ However, because the level of GFAP expression in astrocytes from the grey matter of the asymptomatic spinal cord (the astrocytes surrounding the motor neurons) seems to be low/moderate as detected by traditional histologic techniques, and in the hSOD1 ${ }^{\mathrm{G} 93 \mathrm{~A}}$ the increase in GFAP+ astrocytes is only observed after symptoms onset (ongoing motor neuron degeneration), the GFAP promoter may not be the optimal choice to study the effect of forced astrocyte-specific EAAT2 expression in ALS models. The presence of aberrant mRNA splice variants for EAAT2 may explain the loss of EAAT2 in ALS, although these variants also exist in control individuals. ${ }^{57,58}$ In addition, the toxicity of mutant hSOD1 has been linked to decreased EAAT2, because oxidative reactions triggered by hydrogen peroxide and catalyzed by mutant, but not wild type, SOD1 inactivated the glutamate transporter. ${ }^{59}$

Astrocytes influence the sensitivity of motor neurons to excitotoxicity by yet another mechanism. The calcium permeability of the AMPA receptor is determined by the presence or absence of the GluR2 subunit. The GluR2 subunit produces receptors that are impermeable to calcium. Astrocytes induce the upregulation of the GluR2 subunit in co-cultured motor neurons, thereby protecting them from excitotoxic damage. However, expression of mutant hSOD1 in astrocytes eliminates their GluR2-regulating capacity and increases motor neuron vulnerability to AMPA receptor-mediated excitotoxicity. ${ }^{60} \mathrm{Al}-$ though the role of excitotoxicity in ALS remains under investigation, riluzole, a drug believed to inhibit the glutamatergic system, induces a modest increase in survival in ALS patients. ${ }^{61-64}$ Riluzole is still the only approved treatment for ALS.

\section{Nitric oxide}

In ALS and ALS-animal models, reactive astrocytes upregulate iNOS expression and display makers of oxidative and nitrative stress. ${ }^{22,23,28,29}$ In vitro, there is a differential susceptibility of astrocytes and neurons to nitric oxide and peroxynitrite (formed by the fast reaction of nitric oxide and superoxide radicals) ${ }^{65,66}$ In neurons, nitric oxide induces mitochondrial dysfunction and cell death, whereas astrocytes remain unaffected. The role of nitric oxide production by reactive astrocytes in neuronal survival can be investigated in vitro by selective activation of astrocytes. In particular, after cytokine treatment astrocytes produce nitric oxide, which (along with peroxynitrite) can inhibit components of the mitochondrial respiratory chain in co-cultured cortical neurons ${ }^{67,68}$ and enhance NMDA-induced excitotoxicity. ${ }^{69,70}$ Similarly, the production of nitric oxide by reactive astrocytes is required for the induction of motor neuron apoptosis in a co-culture model where embryonic 
rat spinal cord motor neurons are plated onto peroxynitrite or bacterial lipopolysaccharide (LPS)-treated astrocyte monolayers. ${ }^{71}$ Under controlled conditions, astrocytes provide sufficient trophic support to allow motor neurons to survive and develop extensive neurite processes without addition of neurotrophic factors. In contrast, approximately $40 \%$ of the motor neurons plated on astrocytes pretreated with peroxynitrite or LPS undergo apoptosis. Addition of a cocktail of neurotrophic factors that support motor neuron survival in pure cultures did not prevent motor neuron loss, thus excluding the inhibition of trophic factor production as a mediator of astrocyte toxicity. However, inhibition of iNOS prevented motor neuron loss, supporting the neurotoxic role of nitric oxide production by astrocytes. In addition, culture media from activated astrocytes, as well as spinal cord lysates from symptomatic hSOD $1^{\mathrm{G} 93 \mathrm{~A}}$ mice induced motor neuron apoptosis, but only when incubated with an exogenous nitric oxide source. ${ }^{72}$ Thus, reactive astrocytes in ALS may create the conditions to actively induce motor neuron death and/or contribute to propagate the disease by nitric oxide-dependent mechanisms.

\section{Death receptors}

Three death receptors, tumor necrosis factor- $\alpha$ (TNF)- $\alpha$, p75 neurotrophin receptor (p75 ${ }^{\mathrm{NTR}}$ ), and Fas/ CD95 have been implicated in cell-autonomous and/or nonautonomous aspects of the disease. Under a particular cellular context, activation of a death receptor triggers a specific signaling cascade leading to death of the cell expressing the receptor. ${ }^{73}$

Both TNF $\alpha$ and its pro-apoptotic receptor TNF-R1 mRNA are elevated at late presymptomatic stages of the disease in the lumbar spinal cord of hSOD1 ${ }^{\mathrm{G} 93 \mathrm{~A}}$ mice and continue to increase during the paralytic phase of the disease. ${ }^{74,75}$ Upregulation of TNF- $\alpha$ precedes transcriptional upregulation of other pro-inflammatory genes and temporally correlates with the progression of the disease in hSOD1 ${ }^{\mathrm{G} 93 \mathrm{~A}}$ mice. ${ }^{74}$ Glial cells are a major source of TNF- $\alpha$ in the CNS. Accordingly, increased TNF- $\alpha$ mRNA expression correlates with the onset of astrogliosis in the spinal cord of the low-copy number hSOD $1{ }^{\mathrm{G} 93 \mathrm{~A}}$ mice at 4 months of age. ${ }^{76}$ In the high-copy number $\mathrm{hSDO} 1^{\mathrm{G} 93 \mathrm{~A}}$ mice, lumbar spinal cord motor neurons and astrocytes express high levels of TNF- $\alpha$ well before the onset of symptoms. ${ }^{77}$ In addition, both TNF- $\alpha$ and soluble TNF receptor are elevated in serum of ALS patients. ${ }^{78}$ In mutant hSOD1 mice, the beneficial effects of several pharmacological treatments are correlated with the reduction in TNF- $\alpha$ levels. ${ }^{77,79-81}$ However, genetic ablation of TNF- $\alpha$ has no beneficial effect on survival, motor axon degeneration, or gliosis in two different lines of mutant SOD1 mice. ${ }^{82}$

Spinal motor neurons express p $75^{\mathrm{NTR}}$ during the embryonic period of naturally occurring cell death, but its expression gradually ceases after birth. ${ }^{83}$ Neither TrkA nor $\mathrm{p} 75^{\mathrm{NTR}}$ are expressed by adult motor neurons, but $\mathrm{p} 75^{\mathrm{NTR}}$ is re-expressed after axotomy ${ }^{84-86}$ and in ALS. ${ }^{87,88}$ Degenerating motor neurons in ALS mouse models re-express p $75^{\text {NTR }}, 88,89$ whereas the surrounding reactive astrocytes express nerve growth factor (NGF). ${ }^{72}$ NGF signaling through $\mathrm{p} 75^{\mathrm{NTR}}$, in the absence of TrkA, is capable of inducing cell death of specific neuronal types. ${ }^{90}$ Accordingly, p $75^{\mathrm{NTR}}$-expressing motor neurons undergo apoptosis when cultured on reactive astrocytes secreting NGF or when treated with exogenous NGF in the presence of a low steady-state concentration of nitric oxide. $^{72,91}$ As mentioned before, spinal cord extracts from symptomatic hSOD1 ${ }^{\mathrm{G} 93 \mathrm{~A}}$ (but not age-matched nontransgenic mice) induce apoptosis of motor neurons in the presence of nitric oxide. ${ }^{72}$ Blocking antibodies against NGF or $\mathrm{p} 75^{\mathrm{NTR}}$ (as well as a small nonpeptidyl blocking ligand of $\mathrm{p} 75^{\mathrm{NTR}}$ ) can significantly prevent the apoptotic activity in hSOD1 $1^{\mathrm{G} 93 \mathrm{~A}}$ spinal cord extracts. ${ }^{72,92}$ In addition, NGF signaling through $\mathrm{p} 75^{\mathrm{NTR}}$ triggers the death of motor neurons expressing hSOD $1^{\mathrm{G} 93 \mathrm{~A}}$, even in the absence of an exogenous source of nitric oxide. ${ }^{93}$ However, earlier attempts to modulate p $75^{\mathrm{NTR}}$-mediated neuronal death in ALS models with antisense oligonucleotides or a cyclic decapeptide antagonist of $\mathrm{p} 75^{\mathrm{NTR}}$ produced conflicting results as to the role of $\mathrm{p} 75^{\mathrm{NTR}}$ in disease progression in vivo. ${ }^{94,95}$ Due to the availability of new small nonpeptidyl blocking p $75^{\mathrm{NTR}}$ ligands, revisiting this issue is an exciting next step..$^{92,96}$

Fas and FasL (FasL-Fas ligand) are also expressed by motor neurons during the embryonic period of naturally occurring cell death. Moreover, Fas signaling has been implicated in axotomy-induced motor neuron death, suggesting that this pathway may be activated in the pathological degeneration of motor neurons. ${ }^{97}$ Motor neuron death can be induced in vitro by activation of a motor neuron-specific pathway downstream of Fas involving nitric oxide production (called the Fas/NO pathway). ${ }^{98,99}$ A strong immunoreactivity for FasL is evident in neurons and astrocytes in the lumbar spinal cord of asymptomatic hSOD1 ${ }^{\mathrm{G} 93 \mathrm{~A}}$, as well as in the spinal cord of familial and sporadic ALS cases. ${ }^{77}$ Motor neurons isolated from ALS-linked hSOD1 (G37R, G85R, and G93A) mutant embryos display increased sensitivity to Fas activation and nitric oxide. ${ }^{98}$ This data suggest that nitric oxide or Fas agonist produced by astrocytes might trigger or amplify motor neuron death. The apoptotic pathway seems to be specific for motor neurons where activation of Fas leads to phosphorylation of p38 through Daxx, caspase- 8 activation and the production of nitric oxide by neuronal nitric oxide synthase. ${ }^{99}$ Interestingly, partial loss-of-function mutations for FasL confer a modest extension of survival in hSOD $1{ }^{\mathrm{G} 93 \mathrm{~A}}$ mice. ${ }^{100} \mathrm{In}$ addition, intrathecal infusion of Fas-small interference RNAs in mutant hSOD1 mice significantly reduces the 
pathological activation of caspase-8, p38, neuronal nitric oxide synthase, and prolongs the lifespan. ${ }^{101}$

\section{ALS-linked hSOD1 expressing astrocytes}

Even though the expression of mutant hSOD1 under the control of GFAP promoter causes astrogliosis in vivo, ${ }^{38}$ primary spinal cord astrocytes expressing hSOD $1^{\mathrm{G} 93 \mathrm{~A}}$ are morphologically equivalent to nontransgenic astrocytes, as determined by immunofluorescence for GFAP and $\alpha$-tubulin. In addition, no increase in the amount of GFAP expression is observed. ${ }^{102}$ Thus, in vitro, the expression of mutant hSOD1 alone does not cause the typical astrocyte activation found in ALS or ALS models. However, in contrast to the trophic support provided by nontransgenic astrocytes, $40 \%$ of motor neurons are lost when co-cultured on untreated spinal cord astrocytes isolated from hSOD $1^{\mathrm{G} 93 \mathrm{~A}}$ rats. ${ }^{103}$ This observation was later extended to mouse astrocytes expressing several ALS-linked mutant hSOD1. ${ }^{104,105}$ Importantly, among a variety of non-neuronal cell types, only astrocytes acquire a toxic property due to mutant hSOD1 expression, whereas among a variety of neuronal subtypes, motor neurons are the only ones sensitive to the astrocyte-induced toxicity. ${ }^{104}$ Although, Nagai et al. ${ }^{104}$ claim to have excluded some potential candidates, the identity of the astrocytic factor(s) that mediate this effect remains controversial. Increased nitric oxide production and mitochondrial dysfunction in hSOD ${ }^{\mathrm{G} 93 \mathrm{~A}}$ astrocytes may contribute to the observed toxicity. ${ }^{103,106}$ Despite the major impact on motor neuron survival, microarray analysis detected few differences in the transcriptional profile of nontransgenic and hSOD $1^{\mathrm{G} 93 \mathrm{~A}}$ astrocytes, even when a low-fold cutoff was used. ${ }^{102}$ The most upregulated gene was decorin $(D c n)$, a small multifunctional extracellular proteoglycan. Downregulated genes included the insulin-like growth factor-1 receptor $(I g f-1 r)$ and the RNA binding protein ROD1. Even though, a direct link between these changes and motor neuron toxicity is missing, changes in the expression of $D c n, I g f-1 r$, and $\operatorname{Rodl} l$ were found in the spinal cord of asymptomatic $\mathrm{hSOD} 1^{\mathrm{G} 93 \mathrm{~A}}$ animals before overt neuronal degeneration, suggesting that these changes could influence astrocytemotor neuron interaction in the course of the disease. ${ }^{102}$

\section{ASTROCYTES AS A THERAPEUTIC TARGET IN ALS}

Weather reactive astrogliosis in ALS is detrimental remains an open question, and it could depend on the stage of the disease (onset vs end-stage) as well as the characteristic and environment of a particular motor neuron. However, treatments that delay onset or prolong survival in ALS-models are often associated with decreased reactive astrogliosis. In this context, targeting astrocytes could mean to target the cell per se or develop treatments that decrease the level of astrogliosis. Since the availability of animal ALS models, an extensive effort has been dedicated to find additional therapies for ALS. The following paragraphs review some of the evidence indicating that astrocytes are a potential therapeutic target in ALS.

During development, motor neurons are particular dependent on the trophic support provided by the target, but its dependence on the target diminished with age. However, after stress or injury, adult motor neurons may again become dependent on trophic support from alternative sources, including astrocytes. Impaired neurotrophic support has been proposed as a direct or indirect cause for the motor neuron loss observed in ALS. ${ }^{107}$ Hence, different growth factors, such as glial cell linederived growth factor (GDNF), insulin-like growth factor I (IGF-I), ciliary neurotrophic factor (CNTF), brainderived growth factor (BDNF), and vascular endothelial growth factor (VEGF) ${ }^{108-112}$ have been evaluated in experimental models of ALS. In most cases, a positive effect has been observed in animal models, but they have failed to live up to the expectations in human trials. ${ }^{113-117}$ More efficient delivery methods, such as implantation of pluripotent precursors that are genetically engineered to produce the growth factor are being developed with mixed outcomes. ${ }^{118,119}$ Although, in general, the cell types to which the precursor differentiates is affected by the kind of precursor and several experimental variables, a significant percentage of precursors may differentiate into astrocytes, suggesting that astrocytes are a therapeutic alternative source of trophic support for motor neurons in the adulthood. Moreover, the protective effect of riluzole may also be related to its capacity to increase motor neuron trophic support from astrocytes. ${ }^{120,121} \mathrm{In}$ some cases, increased growth factor availability decreases the number of microglia and reactive astrocytes, suggesting that in addition to the direct neurotrophic activity on motor neurons, growth factor mediated suppression of astrogliosis may participate in the delay of disease progression. ${ }^{122,123}$

Based on the role of astrocytes in maintaining glutamate homeostasis in the CNS, a number of Food and Drug Administration (FDA)-approved drugs were screened for its capacity to modulate glutamate transport. The study identified $\beta$-lactam antibiotics as potent stimulators of GLT1 expression but not GLAST expression. ${ }^{124}$ Treatment with the $\beta$-lactam ceftriaxone increases expression of EAAT2/GLT1 and glutamate uptake in primary human fetal astrocytes and mouse brain. ${ }^{125}$ Ceftriaxone treatment delayed motor neuron and muscle strength loss, and increased survival in an ALS animal model. ${ }^{124}$ This treatment in combination with a glutamate receptor subtype selective drug is a promising strategy to combat excitotoxicity in ALS. In addition, it has been suggested recently that ceftriaxone, 
through activation of antioxidant defenses, is also protective against oxidative glutamate toxicity, a form of glutamate-induced cell death distinct from excitotoxicity. ${ }^{126}$

Prostaglandin synthesis within the CNS is dependent on the catalytic action of COX-2. Levels of COX-2 and its product prostaglandin E2 (PGE2) are increased in hSOD $1^{\mathrm{G} 93 \mathrm{~A}}$ transgenic mice. ${ }^{127,128}$ Increased levels of both COX-2 and PGE2 have been reported in human postmortem spinal cords and in cerebrospinal fluid of sporadic ALS patients. ${ }^{127,129,130}$ Moreover, the E-prostanoid receptor for PGE2 is upregulated in spinal cord astrocytes of symptomatic hSOD $1^{\mathrm{G} 93 \mathrm{~A}}$ mice and ALS patients, ${ }^{131}$ where it seems to participate in the PGE2mediated induction of proinflammatory proteins and the production of free radicals by astrocytes. In addition, the prostaglandin D2 (PGD2) receptor is upregulated more than 14-fold in hSOD1 ${ }^{\mathrm{G} 93 \mathrm{~A}}$-expressing glia and a specific antagonist of the PGD2 receptor partially rescues motor neurons from hSOD $1^{\mathrm{G} 93 \mathrm{~A}}$-glia toxicity. ${ }^{132} \mathrm{COX}$ inhibitors reduce astrocytic glutamate release ${ }^{133}$ and therefore may have a therapeutic effect in ALS. The administration of COX-2 inhibitors ${ }^{128,134,135}$ or the lack of the E-prostanoid receptor ${ }^{131}$ extends the survival in hSOD $1^{\mathrm{G} 93 \mathrm{~A}}$ mice. However, celecoxib, a COX-2 inhibitor approved by the Food and Drug Administration did not have any beneficial effect in ALS patients. ${ }^{136}$

Increased oxidative stress has been implicated in the pathogenesis of ALS and a variety of antioxidants have been tested in ALS patients. ${ }^{137}$ Several studies have demonstrated that by releasing glutathione ( $\gamma$-l-glutamyl-1-cysteinylglycine, [GSH]), astrocytes can improve the antioxidant defenses in co-cultured neurons. Astrocyte-secreted GSH can boost GSH levels in neurons and also act as an antioxidant in the extracellular compartment. ${ }^{138-140}$ In agreement, GSH secretion by astrocytes has been demonstrated to protect neurons against oxidative stress, the deleterious effect of nitric oxide and $\mathrm{p} 75^{\mathrm{NTR}}$-dependent apoptosis. ${ }^{6}$ There are no differences in the amount of GSH produced by hSOD $1^{\mathrm{G} 93 \mathrm{~A}}$ or nontransgenic astrocytes, but increasing GSH release from hSOD1 $1^{\mathrm{G} 93 \mathrm{~A}}$ astrocytes prevents its toxicity toward co-cultured motor neurons. ${ }^{103} \mathrm{GSH}$ is synthesized by the consecutive action of two enzymes: glutamate-cysteine ligase and glutathione synthetase. The transcription factor nuclear factor erythroid-2-related factor 2 (Nrf2) regulates both enzymes, and hence increases GSH production and releases to the extracellular medium on activation. Our laboratory has shown that ALS mice with specific astrocyte Nrf2 overexpression developed the disease later, survived longer, and had lower glial reactivity. ${ }^{141}$ In vitro evidence suggests that increased Nrf2-induced astrocytic GSH secretion could account for the observed motor neuron preservation. ${ }^{141} \mathrm{Nrf} 2$ activation also upregulates heme oxygenase-1, and the induction of heme oxygenase- 1 has been shown to prevent oxidative-mediated damage in nervous cells. Both Nrf2 and heme oxygenase- 1 levels were increased and co-localized with reactive astrocytes in the degenerating lumbar spinal cord of hSOD $1^{\mathrm{G} 93 \mathrm{~A}}$ rats. ${ }^{142}$ These data not only validate Nrf2 as a viable therapeutic target in ALS, but they also stress the role of astrocyte antioxidant defenses in determining motor neuron fate in the disease. In addition, pretreatment of mutant hSOD1 astrocyte monolayers with NOS inhibitors, mitochondrial-targeted antioxidants (ubiquinone and carboxy-proxyl nitroxide) ${ }^{106}$ or NADPH oxidase (NOX2) ${ }^{143,144}$ inhibitors prevented motor neuron loss, suggesting that multiple pathways could be targeted to improve the antioxidant status of the astrocytes and prevent motor neuron death in ALS.

In cell replacement therapies, the intrinsic complications of replacing highly specialized cells, such as motor neurons, make astrocytes a much more appealing target. As mentioned before, in chimeric animals, the degeneration of motor neurons expressing mutant hSOD1 was delayed when surrounded by wild-type non-neuronal cells. ${ }^{43}$ Recently, Lepore et al. ${ }^{145}$ transplanted glial-restricted precursors (GRPs, lineage-restricted astrocyte precursors) into the spinal cord of hSOD $1^{\mathrm{G} 93 \mathrm{~A}}$ rats. Since respiratory failure precipitates death in ALS, transplants were aimed around specific motor neuron pools of the cervical spinal cord involved in respiratory function. GRPs survived in diseased tissue, differentiated efficiently into astrocytes and reduced microgliosis in the cervical spinal cords of hSOD ${ }^{\mathrm{G} 93 \mathrm{~A}}$ rats. GRPs also extended survival and disease duration, attenuated motor neuron loss and slowed declines in forelimb motor and respiratory physiological functions. These results demonstrate that probably, the most straightforward and plausible therapeutic application of astrocytes in ALS is its focal transplantation into regions of the spinal cord in order to protect specific groups of motor neurons. In addition, in the context of a chronic neurodegenerative disease where the newly transplanted-differentiated astrocytes might be adversely affected by the toxic environment, transplantation of modified GRPs (such as Nrf2-overexpressing GRPs) might confer increased protection, and it is an exciting option.

Finally, it may be possible to develop pharmacological and viral approaches to specifically decrease astrogliosis and achieve neuroprotection. Viral delivery of IGF-1 causes a notable extension in the lifespan of ALS animals. ${ }^{109}$ In addition to its direct effect on motor neurons, IGF-1 seems to attenuate gliosis and glial cell-mediated release of TNF $\alpha$ and nitric oxide, thereby decreasing the pathological activity of nonneuronal cells in this model. ${ }^{109,146}$ Interestingly, it might be possible to specifically target astrocytes in adult mice by intravenous injection of specific adenoassociated virus serotypes. ${ }^{147}$ The chemical com- 
pound, (R)-(-)-2-propyloctanoic acid (ONO-2506), an enantiomeric homologue of valproate, restores normal astrocyte functions in a stroke model by preventing reactive astrogliosis. ${ }^{148}$ ONO-2506 causes a pronounced inhibition of S-100 $\beta$ and NGF expression in reactive astrocytes. In addition, it has significant antiglutamate and anti-inflammatory properties, and phase II trials are in progress. ${ }^{137}$ Although the concept of astrocytes as a therapeutic target in neurodegeneration is mainly understood as therapeutic vehicles to prevent motor neuron death, there is evidence of focal degeneration of astrocytes in $\mathrm{hSOD} 1^{\mathrm{G} 93 \mathrm{~A}}$ mice, and its prevention might have beneficial effects in ALS. ${ }^{149}$

It is clear that reactive astrogliosis can no longer be conceived as a passive feature of neurodegeneration. Although normal astrocytes provide an optimized environment to neuronal function, astrocytes in ALS have the potential to be protective and/or toxic to motor neurons. Although further investigation is needed to precisely determine the origin and consequences of the reactive astrogliosis in ALS, recent advances in the involvement of non-neuronal cells in motor neuron pathology provides new therapeutic targets. Due to the complex nature of neuronal biology and the probable multifactorial causes of ALS, the most advantageous therapy will target neuronal, glial, and endothelial cells to effectively protect the whole functional unit. Most likely, non-neuronal cells actively participate in several pathological conditions of the CNS, and therapies targeting astrocytes could contribute to the development of integral approaches to ischemic injury, trauma, and other neurodegenerative diseases.

Acknowledgments: This work was supported by grants from the Amyotrophic Lateral Sclerosis (ALS) Association and Grant No. ES08089 from the National Institute of Environmental Health Sciences (NIEHS) to JAJ. In addition, MRV was supported by the Milton Safenowitz postdoctoral fellowship for ALS research from the ALS Association.

\section{REFERENCES}

1. Oberheim NA, Takano T, Han X, et al. Uniquely hominid features of adult human astrocytes. J Neurosci 2009;29:3276-3287.

2. Eng LF, Ghirnikar RS, Lee YL. Glial fibrillary acidic protein: GFAP-thirty-one years (1969-2000). Neurochem Res 2000;25: 1439-1451.

3. Bushong EA, Martone ME, Jones YZ, Ellisman MH. Protoplasmic astrocytes in CA1 stratum radiatum occupy separate anatomical domains. J Neurosci 2002;22:183-192.

4. Nedergaard M, Ransom B, Goldman SA. New roles for astrocytes: redefining the functional architecture of the brain. Trends Neurosci 2003;26:523-530.

5. Koehler RC, Roman RJ, Harder DR. Astrocytes and the regulation of cerebral blood flow. Trends Neurosci 2009;32:160-169.

6. Vargas MR, Johnson JA. The Nrf2-ARE cytoprotective pathway in astrocytes. Expert Rev Mol Med 2009;11:e17.

7. Perea G, Navarrete M, Araque A. Tripartite synapses: astrocytes process and control synaptic information. Trends Neurosci 2009; $32: 421-431$.
8. Pekny M, Nilsson M. Astrocyte activation and reactive gliosis. Glia 2005;50:427-434.

9. Sofroniew MV. Molecular dissection of reactive astrogliosis and glial scar formation. Trends Neurosci 2009;32:638-647.

10. Eddleston M, Mucke L. Molecular profile of reactive astrocytesimplications for their role in neurologic disease. Neuroscience 1993;54:15-36.

11. Ridet JL, Malhotra SK, Privat A, Gage FH. Reactive astrocytes: cellular and molecular cues to biological function. Trends Neurosci 1997;20:570-577.

12. Buffo A, Rite I, Tripathi P, et al. Origin and progeny of reactive gliosis: a source of multipotent cells in the injured brain. Proc Natl Acad Sci U S A 2008;105:3581-3586.

13. Magnus T, Carmen J, Deleon J, et al. Adult glial precursor proliferation in mutant SOD1G93A mice. Glia 2008;56:200-208.

14. Rowland LP, Shneider NA. Amyotrophic lateral sclerosis. N Engl J Med 2001;344:1688-1700.

15. Rosen DR, Siddique T, Patterson D, et al. Mutations in $\mathrm{Cu} / \mathrm{Zn}$ superoxide dismutase gene are associated with familial amyotrophic lateral sclerosis. Nature 1993;362:59-62.

16. Gurney ME, Pu H, Chiu AY, et al. Motor neuron degeneration in mice that express a human $\mathrm{Cu}, \mathrm{Zn}$ superoxide dismutase mutation. Science 1994;264:1772-1775.

17. Howland DS, Liu J, She Y, et al. Focal loss of the glutamate transporter EAAT2 in a transgenic rat model of SOD1 mutantmediated amyotrophic lateral sclerosis (ALS). Proc Natl Acad Sci U S A 2002;99:1604-1609.

18. Kushner PD, Stephenson DT, Wright S. Reactive astrogliosis is widespread in the subcortical white matter of amyotrophic lateral sclerosis brain. J Neuropathol Exp Neurol 1991;50:263-277.

19. Nagy D, Kato T, Kushner PD. Reactive astrocytes are widespread in the cortical gray matter of amyotrophic lateral sclerosis. J Neurosci Res 1994;38:336-347.

20. O'Reilly SA, Roedica J, Nagy D, et al. Motor neuron-astrocyte interactions and levels of $\mathrm{Cu}, \mathrm{Zn}$ superoxide dismutase in sporadic amyotrophic lateral sclerosis. Exp Neurol 1995;131:203-210.

21. Schiffer D, Cordera S, Cavalla P, Migheli A. Reactive astrogliosis of the spinal cord in amyotrophic lateral sclerosis. J Neurol Sci 1996;139(Suppl):27-33.

22. Schiffer D, Fiano V. Astrogliosis in ALS: possible interpretations according to pathogenetic hypotheses. Amyotroph Lateral Scler Other Motor Neuron Disord 2004;5:22-25.

23. Barbeito LH, Pehar M, Cassina P, et al. A role for astrocytes in motor neuron loss in amyotrophic lateral sclerosis. Brain Res Brain Res Rev 2004;47:263-274.

24. Wong PC, Pardo CA, Borchelt DR, et al. An adverse property of a familial ALS-linked SOD1 mutation causes motor neuron disease characterized by vacuolar degeneration of mitochondria. Neuron 1995;14:1105-1116.

25. Bruijn LI, Becher MW, Lee MK, et al. ALS-linked SOD1 mutant G85R mediates damage to astrocytes and promotes rapidly progressive disease with SOD1-containing inclusions. Neuron 1997; 18:327-338.

26. Hall ED, Oostveen JA, Gurney ME. Relationship of microglial and astrocytic activation to disease onset and progression in a transgenic model of familial ALS. Glia 1998;23:249-256.

27. Levine JB, Kong J, Nadler M, Xu Z. Astrocytes interact intimately with degenerating motor neurons in mouse amyotrophic lateral sclerosis (ALS). Glia1999;28:215-224.

28. Almer G, Vukosavic S, Romero N, Przedborski S. Inducible nitric oxide synthase up-regulation in a transgenic mouse model of familial amyotrophic lateral sclerosis. J Neurochem 1999;72: $2415-2425$.

29. Sasaki S, Warita H, Abe K, Iwata M. Inducible nitric oxide synthase (iNOS) and nitrotyrosine immunoreactivity in the spinal cords of transgenic mice with a G93A mutant SOD1 gene. J Neuropathol Exp Neurol 2001;60:839-846.

30. Wang J, Xu G, Li H, et al. Somatodendritic accumulation of misfolded SOD1-L126Z in motor neurons mediates degeneration: alphaB-crystallin modulates aggregation. Hum Mol Genet 2005; 14:2335-2347.

31. Wang J, Xu G, Gonzales V, et al. Fibrillar inclusions and motor neuron degeneration in transgenic mice expressing superoxide 
dismutase 1 with a disrupted copper-binding site. Neurobiol Dis 2002;10:128-138.

32. Wang J, Slunt H, Gonzales V, et al. Copper-binding-site-null SOD1 causes ALS in transgenic mice: aggregates of non-native SOD1 delineate a common feature. Hum Mol Genet 2003;12: 2753-2764.

33. Nagai M, Aoki M, Miyoshi I, et al. Rats expressing human cytosolic copper-zinc superoxide dismutase transgenes with amyotrophic lateral sclerosis: associated mutations develop motor neuron disease. J Neurosci 2001;21:9246-9254.

34. Beckman JS, Estevez AG, Crow JP, Barbeito L. Superoxide dismutase and the death of motoneurons in ALS. Trends Neurosci 2001;24(11 Suppl):S15-20.

35. Cleveland DW, Rothstein JD. From Charcot to Lou Gehrig: deciphering selective motor neuron death in ALS. Nature Rev Neurosci 2001;2:806-819.

36. Bruijn LI, Miller TM, Cleveland DW. Unraveling the mechanisms involved in motor neuron degeneration in ALS. Annu Rev Neurosci 2004;27:723-749.

37. Manfredi G, Xu Z. Mitochondrial dysfunction and its role in motor neuron degeneration in ALS. Mitochondrion 2005;5:7787.

38. Gong YH, Parsadanian AS, Andreeva A, Snider WD, Elliott JL. Restricted expression of $\mathrm{G} 86 \mathrm{R} \mathrm{Cu} / \mathrm{Zn}$ superoxide dismutase in astrocytes results in astrocytosis but does not cause motoneuron degeneration. J Neurosci 2000;20:660-665.

39. Pramatarova A, Laganière J, Roussel J, Brisebois K, Rouleau GA. Neuron-specific expression of mutant superoxide dismutase 1 in transgenic mice does not lead to motor impairment. J Neurosci 2001;21:3369-3374.

40. Lino MM, Schneider C, Caroni P. Accumulation of SOD1 mutants in postnatal motoneurons does not cause motoneuron pathology or motoneuron disease. J Neurosci 2002;22:4825-4832.

41. Jaarsma D, Teuling E, Haasdijk ED, De Zeeuw CI, Hoogenraad CC. Neuron-specific expression of mutant superoxide dismutase is sufficient to induce amyotrophic lateral sclerosis in transgenic mice. J Neurosci 2008;28:2075-2088.

42. Lepore AC, Dejea C, Carmen J, et al. Selective ablation of proliferating astrocytes does not affect disease outcome in either acute or chronic models of motor neuron degeneration. Exp Neurol 2008;211:423-432.

43. Clement AM, Nguyen MD, Roberts EA, et al. Wild-type nonneuronal cells extend survival of SOD1 mutant motor neurons in ALS mice. Science 2003;302:113-117.

44. Boillée S, Yamanaka K, Lobsiger CS, et al. Onset and progression in inherited ALS determined by motor neurons and microglia. Science 2006;312:1389-1392.

45. Yamanaka K, Boillee S, Roberts EA, et al. Mutant SOD1 in cell types other than motor neurons and oligodendrocytes accelerates onset of disease in ALS mice. Proc Natl Acad Sci U S A 2008; 105:7594-7599.

46. Wang L, Sharma K, Grisotti G, Roos RP. The effect of mutant SOD1 dismutase activity on non-cell autonomous degeneration in familial amyotrophic lateral sclerosis. Neurobiol Dis 2009;35: 234-240.

47. Beers DR, Henkel JS, Xiao Q, et al. Wild-type microglia extend survival in PU.1 knockout mice with familial amyotrophic lateral sclerosis. Proc Natl Acad Sci U S A 2006;103:16021-16026.

48. Yamanaka K, Chun SJ, Boillee S, et al. Astrocytes as determinants of disease progression in inherited amyotrophic lateral sclerosis. Nat Neurosci 2008;11:251-253.

49. Boillée S, Vande Velde C, Cleveland DW. ALS: a disease of motor neurons and their nonneuronal neighbors. Neuron 2006; $52: 39-59$.

50. Lobsiger CS, Cleveland DW. Glial cells as intrinsic components of non-cell-autonomous neurodegenerative disease. Nat Neurosci 2007;10:1355-1360.

51. Ilieva H, Polymenidou M, Cleveland DW. Non-cell autonomous toxicity in neurodegenerative disorders: ALS and beyond. J Cell Biol 2009;187:761-772.

52. Rothstein JD, Tsai G, Kuncl RW, et al. Abnormal excitatory amino acid metabolism in amyotrophic lateral sclerosis. Ann Neurol 1990;28:18-25.
53. Spreux-Varoquaux O, Bensimon G, Lacomblez L, et al. Glutamate levels in cerebrospinal fluid in amyotrophic lateral sclerosis: a reappraisal using a new HPLC method with coulometric detection in a large cohort of patients. J Neurol Sci 2002;193:73-78.

54. Rothstein JD, Van Kammen M, Levey AI, Martin LJ, Kuncl RW. Selective loss of glial glutamate transporter GLT-1 in amyotrophic lateral sclerosis. Ann Neurol 1995;38:73-84.

55. Bendotti C, Tortarolo M, Suchak SK, et al. Transgenic SOD1 G93A mice develop reduced GLT-1 in spinal cord without alterations in cerebrospinal fluid glutamate levels. J Neurochem 2001; 79:737-746.

56. Guo H, Lai L, Butchbach ME, et al. Increased expression of the glial glutamate transporter EAAT2 modulates excitotoxicity and delays the onset but not the outcome of ALS in mice. Hum Mol Genet 2003;12:2519-2532.

57. Lin CL, Bristol LA, Jin L, et al. Aberrant RNA processing in a neurodegenerative disease: the cause for absent EAAT2, a glutamate transporter, in amyotrophic lateral sclerosis. Neuron 1998; 20:589-602

58. Meyer T, Fromm A, Münch C, et al. The RNA of the glutamate transporter EAAT2 is variably spliced in amyotrophic lateral sclerosis and normal individuals. J Neurol Sci 1999;170:45-50.

59. Trotti D, Rolfs A, Danbolt NC, Brown RH Jr, Hediger MA SOD1 mutants linked to amyotrophic lateral sclerosis selectively inactivate a glial glutamate transporter. Nat Neurosci 1999;2: 427-433.

60. Van Damme P, Bogaert E, Dewil M, et al. Astrocytes regulate GluR2 expression in motor neurons and their vulnerability to excitotoxicity. Proc Natl Acad Sci U S A 2007;104:14825-14830.

61. Bensimon G, Lacomblez L, Meininger V. A controlled trial of riluzole in amyotrophic lateral sclerosis. ALS/Riluzole Study Group. N Engl J Med 1994;330:585-591.

62. Lacomblez L, Bensimon G, Leigh PN, et al. A confirmatory dose-ranging study of riluzole in ALS. ALS/Riluzole Study Group-II. Neurology 1996;47:S242-S250.

63. Miller RG, Bouchard JP, Duquette P, et al. Clinical trials of riluzole in patients with ALS. ALS/Riluzole Study Group-II. Neurology 1996;47:S86-S90.

64. Lacomblez L, Bensimon G, Leigh PN, Guillet P, Meininger V. Dose-ranging study of riluzole in amyotrophic lateral sclerosis. Amyotrophic Lateral Sclerosis/Riluzole Study Group II. Lancet 1996;347:1425-1431.

65. Bolaños JP, Heales SJ, Land JM, Clark JB. Effect of peroxynitrite on the mitochondrial respiratory chain: differential susceptibility of neurones and astrocytes in primary culture. J Neurochem 1995; 64:1965-1972.

66. Almeida A, Almeida J, Bolaños JP, Moncada S. Different responses of astrocytes and neurons to nitric oxide: the role of glycolytically generated ATP in astrocyte protection. Proc Natl Acad Sci U S A 2001;98:15294-15299.

67. Stewart VC, Sharpe MA, Clark JB, Heales SJ. Astrocyte-derived nitric oxide causes both reversible and irreversible damage to the neuronal mitochondrial respiratory chain. J Neurochem 2000;75: 694-700.

68. Stewart VC, Land JM, Clark JB, Heales SJ. Pretreatment of astrocytes with interferon-alpha/beta prevents neuronal mitochondrial respiratory chain damage. J Neurochem 1998;70:432434.

69. Hewett SJ, Csernansky CA, Choi DW. Selective potentiation of NMDA-induced neuronal injury following induction of astrocytic iNOS. Neuron 1994;13:487-494.

70. Stewart VC, Heslegrave AJ, Brown GC, Clark JB, Heales SJ Nitric oxide-dependent damage to neuronal mitochondria involves the NMDA receptor. Eur J Neurosci 2002;15:458-464.

71. Cassina P, Peluffo H, Pehar M, et al. Peroxynitrite triggers a phenotypic transformation in spinal cord astrocytes that induces motor neuron apoptosis. J Neurosci Res 2002;67:21-29.

72. Pehar M, Cassina P, Vargas MR, et al. Astrocytic production of nerve growth factor in motor neuron apoptosis: implications for amyotrophic lateral sclerosis. J Neurochem 2004;89:464-473.

73. Haase G, Pettmann B, Raoul C, Henderson CE. Signaling by death receptors in the nervous system. Curr Opin Neurobiol 2008; 18:284-291. 
74. Hensley K, Floyd RA, Gordon B, et al. Temporal patterns of cytokine and apoptosis-related gene expression in spinal cords of the G93A-SOD1 mouse model of amyotrophic lateral sclerosis. J Neurochem 2002;82:365-374.

75. Veglianese P, Lo Coco D, Bao Cutrona M, et al. Activation of the p38MAPK cascade is associated with upregulation of TNF alpha receptors in the spinal motor neurons of mouse models of familial ALS. Mol Cell Neurosci 2006;31:218-231.

76. Elliott JL. Cytokine upregulation in a murine model of familial amyotrophic lateral sclerosis. Brain Res Mol Brain Res 2001;95: 172-178.

77. Kiaei M, Petri S, Kipiani K, et al. Thalidomide and lenalidomide extend survival in a transgenic mouse model of amyotrophic lateral sclerosis. J Neurosci 2006;26:2467-2473.

78. Poloni M, Facchetti D, Mai R, et al. Circulating levels of tumour necrosis factor and its soluble receptors are increased in the blood of patients with amyotrophic lateral sclerosis. Neurosci Lett 2000; 287:211-214.

79. Kiaei M, Kipiani K, Calingasan NY, et al. Matrix metalloproteinase-9 regulates TNF-alpha and FasL expression in neuronal, glial cells and its absence extends life in a transgenic mouse model of amyotrophic lateral sclerosis. Exp Neurol 2007;205:7481.

80. Petri S, Calingasan NY, Alsaied OA, et al. The lipophilic metal chelators DP-109 and DP-460 are neuroprotective in a transgenic mouse model of amyotrophic lateral sclerosis. J Neurochem 2007;102:991-1000.

81. Neymotin A, Petri S, Calingasan NY, et al. Lenalidomide (Revlimid) administration at symptom onset is neuroprotective in a mouse model of amyotrophic lateral sclerosis. Exp Neurol 2009; 220:191-197.

82. Gowing G, Dequen F, Soucy G, Julien JP. Absence of tumor necrosis factor-alpha does not affect motor neuron disease caused by superoxide dismutase 1 mutations. J Neurosci 2006;26:1139711402 .

83. Yan Q, Johnson EM Jr. An immunohistochemical study of the nerve growth factor receptor in developing rats. J Neurosci 1988; 8:3481-3498.

84. Koliatsos VE, Crawford TO, Price DL. Axotomy induces nerve growth factor receptor immunoreactivity in spinal motor neurons. Brain Res 1991;549:297-304.

85. Rende M, Giambanco I, Buratta M, Tonali P. Axotomy induces a different modulation of both low-affinity nerve growth factor receptor and choline acetyltransferase between adult rat spinal and brainstem motoneurons. J Comp Neurol 1995;363:249-263.

86. Ferri CC, Moore FA, Bisby MA. Effects of facial nerve injury on mouse motoneurons lacking the p75 low-affinity neurotrophin receptor. J. Neurobiol 1998;34:1-9.

87. Seeburger JL, Tarras S, Natter H, Springer JE. Spinal cord motoneurons express p75NGFR and p145trkB mRNA in amyotrophic lateral sclerosis. Brain Res 1993;621:111-115.

88. Lowry KS, Murray SS, McLean CA, et al. A potential role for the p75 low-affinity neurotrophin receptor in spinal motor neuron degeneration in murine and human amyotrophic lateral sclerosis. Amyotroph Lateral Scler Other Motor Neuron Disord 2001;2: 127-134.

89. Copray JC, Jaarsma D, Kust BM, et al. Expression of the low affinity neurotrophin receptor p75 in spinal motoneurons in a transgenic mouse model for amyotrophic lateral sclerosis. Neuroscience 2003;116: 685-694.

90. Frade JM, Rodríguez-Tébar A, Barde YA. Induction of cell death by endogenous nerve growth factor through its p75 receptor. Nature 1996;383:166-168.

91. Cassina P, Pehar M, Vargas MR, et al. Astrocyte activation by fibroblast growthfactor-1 and motor neuron apoptosis: implications for amyotrophic lateral sclerosis. J Neurochem 2005;93:3846.

92. Pehar M, Cassina P, Vargas MR, et al. Modulation of p75dependent motor neuron death by a small non-peptidyl mimetic of the neurotrophin loop 1 domain. Eur J Neurosci 2006;24:15751580.

93. Pehar M, Vargas MR, Robinson KM, et al. Mitochondrial superoxide production and nuclear factor erythroid 2-related factor 2 activation in p75 neurotrophin receptor-induced motor neuron apoptosis. J Neurosci 2007;27:7777-7785.

94. Turner BJ, Cheah IK, Macfarlane KJ, et al. Antisense peptide nucleic acid-mediated knockdown of the p75 neurotrophin receptor delays motor neuron disease in mutant SOD1 transgenic mice. J Neurochem 2003;87:752-763.

95. Turner BJ, Murray SS, Piccenna LG, Lopes EC, Kilpatrick TJ, Cheema SS. Effect of p75 neurotrophin receptor antagonist on disease progression in transgenic amyotrophic lateral sclerosis mice. J Neurosci Res 2004;78:193-199.

96. Massa SM, Xie Y, Yang T, et al. Small, nonpeptide p75NTR ligands induce survival signaling and inhibit proNGF-induced death. J Neurosci 2006;26:5288-5300.

97. Ugolini G, Raoul C, Ferri A, et al. Fas/tumor necrosis factor receptor death signaling is required for axotomy-induced death of motoneurons in vivo. J Neurosci 2003;23:8526-8531.

98. Raoul C, Estévez AG, Nishimune H, et al. Motoneuron death triggered by a specific pathway downstream of Fas. potentiation by ALS-linked SOD1 mutations. Neuron 2002;35:1067-1083.

99. Raoul C, Buhler E, Sadeghi C, et al. Chronic activation in presymptomatic amyotrophic lateral sclerosis (ALS) mice of a feedback loop involving Fas, Daxx, and FasL. Proc Natl Acad Sci U S A 2006;103:6007-6012.

100. Petri S, Kiaei M, Wille E, Calingasan NY, Beal MF. Loss of Fas ligand-function improves survival in G93A-transgenic ALS mice. J Neurol Sci 2006;251:44-49.

101. Locatelli F, Corti S, Papadimitriou D, et al. Fas small interfering RNA reduces motoneuron death in amyotrophic lateral sclerosis mice. Ann Neurol 2007;62:81-92.

102. Vargas MR, Pehar M, Díaz-Amarilla PJ, Beckman JS, Barbeito L. Transcriptional profile of primary astrocytes expressing ALSlinked mutant SOD1. J Neurosci Res 2008;86:3515-3525.

103. Vargas MR, Pehar M, Cassina P, Beckman JS, Barbeito L. Increased glutathione biosynthesis by Nrf2 activation in astrocytes prevents p75NTR-dependent motor neuron apoptosis. J Neurochem 2006;97:687-696.

104. Nagai M, Re DB, Nagata T, et al. Astrocytes expressing ALSlinked mutated SOD1 release factors selectively toxic to motor neurons. Nat Neurosci 2007;10:615-622.

105. Di Giorgio FP, Carrasco MA, Siao MC, Maniatis T, Eggan K. Non-cell autonomous effect of glia on motor neurons in an embryonic stem cell-based ALS model. Nat Neurosci 2007;10:608614.

106. Cassina P, Cassina A, Pehar M, et al. Mitochondrial dysfunction in SOD1G93A-bearing astrocytes promotes motor neuron degeneration: prevention by mitochondrial-targeted antioxidants. J Neurosci 2008;28:4115-4122.

107. Ekestern E. Neurotrophic factors and amyotrophic lateral sclerosis. Neurodegener Dis 2004;1:88-100.

108. Acsadi G, Anguelov RA, Yang H, et al. Increased survival and function of SOD1 mice after glial cell-derived neurotrophic factor gene therapy. Hum Gene Ther 2002;13:1047-1059.

109. Kaspar BK, Lladó J, Sherkat N, Rothstein JD, Gage FH. Retrograde viral delivery of IGF-1 prolongs survival in a mouse ALS model. Science 2003;301:839-842.

110. Sagot Y, Tan SA, Baetge E, Schmalbruch H, Kato AC, Aebischer P. Polymer encapsulated cell lines genetically engineered to release ciliary neurotrophic factor can slow down progressive motor neuronopathy in the mouse. Eur J Neurosci 1995;7:1313-1322.

111. Azzouz M, Ralph GS, Storkebaum E, et al. VEGF delivery with retrogradely transported lentivector prolongs survival in a mouse ALS model. Nature 2004;429:413-417.

112. Barbeito AG, Martinez-Palma L, Vargas MR, et al. Lead exposure stimulates VEGF expression in the spinal cord and extends survival in a mouse model of ALS. Neurobiol Dis 2010;37:574 580.

113. Borasio GD, Robberecht W, Leigh PN, et al. A placebo-controlled trial of insulin-like growth factor-I in amyotrophic lateral sclerosis. European ALS/IGF-I Study Group. Neurology 1998; 51:583-586.

114. Lai EC, Felice KJ, Festoff BW, et al. Effect of recombinant human insulin-like growth factor-I on progression of ALS. A 
placebo-controlled study. The North America ALS/IGF-I Study Group. Neurology 1997;49:1621-1630.

115. [No authors listed]. A controlled trial of recombinant methionyl human BDNF in ALS: The BDNF Study Group (Phase III). Neurology 1999;52:1427-1433.

116. [No authors listed]. A double-blind placebo-controlled clinical trial of subcutaneous recombinant human ciliary neurotrophic factor (rHCNTF) in amyotrophic lateral sclerosis. ALS CNTF Treatment Study Group. Neurology 1996;46:1244-1249.

117. Bongioanni P, Reali C, Sogos V. Ciliary neurotrophic factor (CNTF) for amyotrophic lateral sclerosis/motor neuron disease. Cochrane Database Syst Rev 2004;3:CD004302.

118. Suzuki M, Svendsen CN. Combining growth factor and stem cell therapy for amyotrophic lateral sclerosis. Trends Neurosci 2008; 31:192-198.

119. Park S, Kim HT, Yun S, et al. Growth factor-expressing human neural progenitor cell grafts protect motor neurons but do not ameliorate motor performance and survival in ALS mice. Exp Mol Med 2009;41:487-500.

120. Peluffo H, Estevez A, Barbeito L, Stutzmann JM. Riluzole promotes survival of rat motoneurons in vitro by stimulating trophic activity produced by spinal astrocyte monolayers. Neurosci Lett 1997;228:207-211.

121. Mizuta I, Ohta M, Ohta K, Nishimura M, Mizuta E, Kuno S. Riluzole stimulates nerve growth factor, brain-derived neurotrophic factor and glial cell line-derived neurotrophic factor synthesis in cultured mouse astrocytes. Neurosci Lett 2001;310:117120.

122. Zheng C, Sköld MK, Li J, Nennesmo I, Fadeel B, Henter JI. VEGF reduces astrogliosis and preserves neuromuscular junctions in ALS transgenic mice. Biochem Biophys Res Commun 2007;363:989-993.

123. Sun W, Funakoshi H, Nakamura T. Overexpression of HGF retards disease progression and prolongs life span in a transgenic mouse model of ALS. J Neurosci 2002;22:6537-6548.

124. Rothstein JD, Patel S, Regan MR, et al. Beta-lactam antibiotics offer neuroprotection by increasing glutamate transporter expression. Nature 2005;433:73-77.

125. Lee SG, Su ZZ, Emdad L, et al. Mechanism of ceftriaxone induction of excitatory amino acid transporter-2 expression and glutamate uptake in primary human astrocytes. J Biol Chem 2008;283:13116-13123.

126. Lewerenz J, Albrecht P, Tien ML, et al. A. Induction of Nrf2 and $\mathrm{xCT}$ are involved in the action of the neuroprotective antibiotic ceftriaxone in vitro. J Neurochem 2009;111:332-343.

127. Almer G, Guégan C, Teismann P, et al. Increased expression of the pro-inflammatory enzyme cyclooxygenase-2 in amyotrophic lateral sclerosis. Ann Neurol 2001;49:176-185.

128. Klivenyi P, Kiaei M, Gardian G, Calingasan NY, Beal MF. Additive neuroprotective effects of creatine and cyclooxygenase 2 inhibitors in a transgenic mouse model of amyotrophic lateral sclerosis. J Neurochem 2004;88:576-582.

129. Yasojima K, Tourtellotte WW, McGeer EG, McGeer PL. Marked increase in cyclooxygenase-2 in ALS spinal cord: implications for therapy. Neurology 2001;57:952-956.

130. Almer G, Teismann P, Stevic Z, et al. Increased levels of the pro-inflammatory prostaglandin PGE2 in CSF from ALS patients. Neurology 2002;58:1277-1279.

131. Liang X, Wang Q, Shi J, et al. The prostaglandin E2 E-prostanoid receptor receptor accelerates disease progression and inflammation in a model of amyotrophic lateral sclerosis. Ann Neurol 2008;64:304-314.

132. Di Giorgio FP, Boulting GL, Bobrowicz S, Eggan KC. Human embryonic stem cell-derived motor neurons are sensitive to the toxic effect of glial cells carrying an ALS-causing mutation. Cell Stem Cell 2008;3:637-648.

133. Bezzi P, Carmignoto G, Pasti L, et al. Prostaglandins stimulate calcium-dependent glutamate release in astrocytes. Nature 1998; 391:281-285.

134. Drachman DB, Frank K, Dykes-Hoberg M, et al. Cyclooxygenase 2 inhibition protects motor neurons and prolongs survival in a transgenic mouse model of ALS. Ann Neurol 2002;52:771-778.

135. Pompl PN, Ho L, Bianchi M, McManus T, Qin W, Pasinetti GM. A therapeutic role for cyclooxygenase- 2 inhibitors in a transgenic mouse model of amyotrophic lateral sclerosis. FASEB J 2003; 17:725-727.

136. Cudkowicz ME, Shefner JM, Schoenfeld DA, et al. Trial of celecoxib in amyotrophic lateral sclerosis. Ann Neurol 2006;60: $22-31$.

137. Traynor BJ, Bruijn L, Conwit R, et al. Neuroprotective agents for clinical trials in ALS: a systematic assessment. Neurology 2006; 67:20-27.

138. Dringen R, Pfeiffer B, Hamprecht B. Synthesis of the antioxidant glutathione in neurons: supply by astrocytes of CysGly as precursor for neuronal glutathione. J Neurosci 1999;19:562-569.

139. Dringen R, Gutterer JM, Hirrlinger J. Glutathione metabolism in brain metabolic interaction between astrocytes and neurons in the defense against reactive oxygen species. Eur J Biochem 2000; 267:4912-4916.

140. Dringen R, Gutterer JM, Gros C, Hirrlinger J. Aminopeptidase N mediates the utilization of the GSH precursor CysGly by cultured neurons. J Neurosci Res 2001;66:1003-1008.

141. Vargas MR, Johnson DA, Sirkis DW, Messing A, Johnson JA. Nrf2 activation in astrocytes protects against neurodegeneration in mouse models of familial amyotrophic lateral sclerosis. J Neurosci 2008;28:13574-13581.

142. Vargas MR, Pehar M, Cassina P, et al. Fibroblast growth factor-1 induces heme oxygenase-1 via nuclear factor erythroid 2-related factor 2 (Nrf2) in spinal cord astrocytes: consequences for motor neuron survival. J Biol Chem 2005;280:25571-25579.

143. Marchetto MC, Muotri AR, Mu Y, Smith AM, Cezar GG, Gage FH. Non-cell-autonomous effect of human SOD1 G37R astrocytes on motor neurons derived from human embryonic stem cells. Cell Stem Cell 2008;3:649-657.

144. Harraz MM, Marden JJ, Zhou W, et al. SOD1 mutations disrupt redox-sensitive Rac regulation of NADPH oxidase in a familial ALS model. J Clin Invest 2008;118:659-670.

145. Lepore AC, Rauck B, Dejea C, et al. Focal transplantation-based astrocyte replacement is neuroprotective in a model of motor neuron disease. Nat Neurosci 2008;11:1294-1301.

146. Dodge JC, Haidet AM, Yang W, et al. Delivery of AAV-IGF-1 to the CNS extends survival in ALS mice through modification of aberrant glial cell activity. Mol Ther 2008;16:1056-1064.

147. Foust KD, Nurre E, Montgomery CL, Hernandez A, Chan CM, Kaspar BK. Intravascular AAV9 preferentially targets neonatal neurons and adult astrocytes. Nat Biotechnol 2009;27:59-65.

148. Tateishi N, Mori T, Kagamiishi Y, et al. Astrocytic activation and delayed infarct expansion after permanent focal ischemia in rats. Part II: suppression of astrocytic activation by a novel agent (R)-(-)-2-propyloctanoic acid (ONO-2506) leads to mitigation of delayed infarct expansion and early improvement of neurologic deficits. J Cereb Blood Flow Metab 2002;22:723-734.

149. Rossi D, Brambilla L, Valori CF, et al. Focal degeneration of astrocytes in amyotrophic lateral sclerosis. Cell Death Differ 2008; 15:1691-700. 